\title{
Les décès toxiques au Maroc : bilan de 5 ans d'étude (1999-2003)
}

\author{
Toxic deaths in Morocco: 5 years \\ assessment of study (1999-2003)
}

\section{Mina AIT EL CADI*, Youssef KHABBAL, Naima MAHNINE, Laarbi IDRISSI}

Département de Toxicologie, Hydrologie et Hygiène Environnementale, Institut National d'Hygiène, 27, avenue Ibn Batouta - BP 769 Rabat - Maroc

*Auteur à qui adresser la correspondance : Dr Mina AIT EL CADI, Département de Toxicologie, Hydrologie et Hygiène Environnementale, Institut National d'Hygiène, 27, avenue Ibn Batouta - BP 769 Rabat - Maroc Tél : +21266089108/+21237 7719 02/+21237771965 - Fax : +21237772067/+21237681653

E-mail : macadiph@yahoo.fr

(Reçu le 25 septembre 2007 ; accepté après modification le 21 décembre 2007)

\section{RÉSUMÉ}

Les décès toxiques sont de différentes origines, les produits incriminés sont aussi de différentes natures, ils occupent une place importante dans l'épidémiologie des mortalités au Maroc. L'objectif de notre travail est de présenter les caractéristiques et décrire la cartographie des décès toxiques au Maroc en se basant sur les résultats d'analyse du laboratoire de toxicologie médicolégale à l'Institut National d'Hygiène. Une étude rétrospective étalée sur 5 ans (janvier. 1999 jusqu'à décembre 2003). Des prélèvements de sang et du contenu gastrique sont analysés à l'aide de réactions colorées, des méthodes chromatographiques (CCM, HPLC, $C P G$ ), la spectrophotométrie de masse et l'absorption atomique. Durant la période entre 1999-2003, 3536 prélèvements médicolégaux sont reçu au laboratoire dont 210 étaient positifs soit 5,9\%. Les intoxications mortelles pendant la durée de notre étude étaient reportées à des intoxications accidentelles dans 46 cas $(21,9 \%)$, aux suicides dans 52 cas $(24,2 \%)$. Les intoxications dont l'origine est indéterminée représentent 109 cas $(51,9 \%)$. La voie orale est la voie la plus rencontrée dans ces intoxications mortelles avec 108

\section{SUMMARY}

The toxic deaths had various origins, the accused products had also various natures, and they occupy an important place in the epidemiology of mortalities in Morocco. The aim of our study is to show the characteristics and to describe the cartography of the toxic deaths in Morocco while being based on the results of the laboratory's analysis of forensic toxicology in the National Institute of Health. A retrospective Study spread out over 5 years (January 1999 until December 2003). Samples of blood and gastric contents are analyzed using coloured reactions, chromatographic methods (CCM, HPLC and CPG), mass spectrophotometer and atomic absorption. Between 1999-2003, 3536 forensic analysis were received at the laboratory of which 210 were positive $(5.9 \%)$. The mortal intoxications throughout our study were accidental in 46 cases $(21.9 \%)$, the suicides was notified in 52 cases (24.2\%), the unspecified origin's intoxications represent 109 cases $(51.9 \%)$. The oral way was most rout in 108 cases (51.4\%) followed by respiratory tract in 30 cases (14.3\%). a highest percentage of mortal intoxication was noted in Rabat with 89 cases $(42.4 \%)$, followed 
cas (51,4\%) suivie de la voie respiratoire dans 30 cas (14,3\%). Un pourcentage élevé dans la ville de Rabat avec 89 cas $(42,4 \%)$, suivie de Casablanca avec 23 cas (11\%), puis Kenitra avec 16 cas $(7,6 \%)$. La mortalité dans notre série est élevée quand il s'agit du paraphényldiamine qui vient en tête avec 44 cas $(21,3 \%)$ suivi du monoxyde de carbone avec 35 cas $(16,9 \%)$ et des pesticides 32 cas $(15,5 \%)$ puis le phostoxin avec 20 cas $(9,7 \%)$. Les décès toxiques au Maroc occupent une place non négligeable dans l'ensemble des décès. Cependant, leur nombre reste sous estimé pour plusieurs raisons, notamment le manque d'informations sur les cas de décès toxiques dans les zones rurales. Des efforts restent à foumir par les parties concernées pour déterminer la part exacte des décès toxiques dans l'épidémiologie des mortalités au Maroc et pour l'instauration d'un registre de décès.

\section{MOTS-CLÉS}

Décès toxiques, monoxyde de carbone, pesticides, phostoxin, paraphényldiamine, intoxications accidentelles.

\section{Introduction}

Les décès par intoxication occupent une place importante dans l'épidémiologie des mortalités au Maroc. La moyenne des intoxications atteint les 700 cas par mois avec un maximum en été entre juillet et août. Les décès toxiques sont de différentes origines, les produits incriminés sont aussi de différentes natures. Les plantes occupent une place importante. En effet, le Maroc est riche en plantes thérapeutiques et toxiques, presque 1039 espèces sont utilisées en pharmacologie (1).

L'utilisation des plantes dans la médecine et dans l'alimentation est fréquente.

L'objectif de notre travail est de présenter les caractéristiques et décrire la cartographie des décès toxiques au Maroc en se basant sur les résultats d'analyse du laboratoire de Toxicologie médicolégale à l'Institut National d'Hygiène sur une période de 5 ans.

\section{Matériel et méthodes}

Le Laboratoire de Toxicologie à l'Institut National d'Hygiène est l'organisme de référence dans les recherches toxicologiques médicolégales. Le laboratoire reçoit les prélèvements provenant des différentes régions du Maroc dans le cadre des autopsies réalisées en cas de mort suspecte. Les paramètres recherchés sont orientés par l'épidémiologie des intoxications.

Le travail présente une étude rétrospective étalée sur 5 ans (janvier 1999 jusqu'à décembre 2003). Les prélèvements standards sont le sang cardiaque et périphérique et le contenu gastrique. Les analyses sont réalisées en routine à l'aide de réactions colorées, des méthodes chromatographiques (CCM, HPLC, CPG) by Casablanca with 23 case (11\%), then Kenitra with 16 case $(7.6 \%)$. Paraphenylendiamine comes at the head with 44 cases (21.3\%) followed by carbon monoxide with 35 cases $(16.9 \%)$, pesticide with 32 cases (15.5\%) then the phostoxin with 20 cases (9.7\%). The toxic deaths in Morocco occupy a considerable place in the whole of the deaths. However; their number remains under estimated for several reasons, in particular lack of information about toxic deaths in a rural area. Efforts remain to be providied by the concerned authority for determine the exact part of the toxic deaths in the mortalities epidemiology in Morocco and for established the death register:

\section{KEY-WORDS}

Toxic death, charbon monoxide, pesticides, phostoxin, paraphényldiamine, accidental intoxications.

couplées ou non à la spectrophotométrie de masse, et l'absorption atomique. Nous avons classé les toxiques recherchés en 7 classes (les gaz toxiques, les pesticides, les médicaments, les raticides, les solvants, les plantes et les déchets industriels)

Les décès toxiques sont classés selon les classifications internationales des maladies [ICD-9] en intoxications suicidaires E950, accidentelles E859 et non déterminées E 980. Les paramètres analysés sont : l’âge, le sexe, la ville, le toxique incriminé et la cause d'intoxication.

Une étude descriptive est réalisée, les résultats sont présentés par la moyenne et l'écart type pour les paramètres quantitatifs.

\section{Résultats}

Durant la période entre 1999-2003, 3536 prélèvements médicolégaux sont reçu au laboratoire dont 210 étaient positifs soit $5,9 \%$ avec un minimum de 554 cas en 2002 soit un pourcentage de positivité de $1,3 \%$ et un maximum de 847 cas en 1999 avec un pourcentage de positivité de $1,72 \%$ (Figure 1 ).

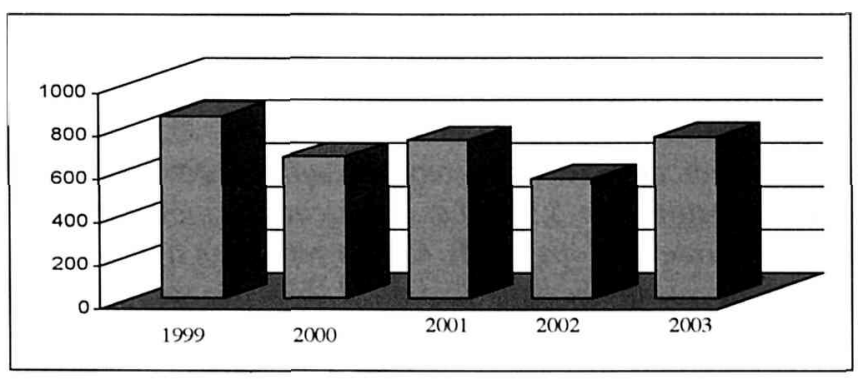

Figure 1 : Nombre de prélèvements reçus par année. 
Les intoxications mortelles pendant la durée de notre étude étaient reportées à des intoxications accidentelles dans 46 cas $(21,9 \%)$, aux suicides dans 52 cas $(24,2 \%)$, les intoxications dont l'origine est indéterminée représentent 109 cas $(51,9 \%)$ (Figure 2).

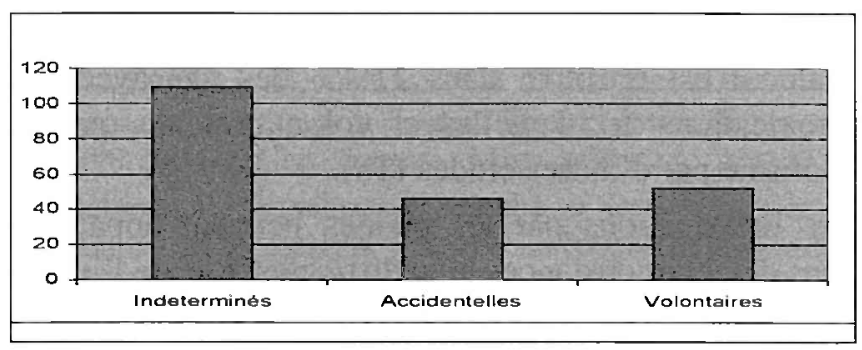

Figure 2 : Fréquence des intoxications mortelles en fonction de leur origine.

La voie orale est la voie la plus rencontrée dans ces intoxications mortelles avec 108 cas $(51,4 \%)$ suivie de la voie respiratoire dans 30 cas $(14,3 \%)$. 68 cas $(32,4 \%)$ n'étaient pas déterminés alors que la voie injectable a été représentée dans un seul cas (Figure 3).

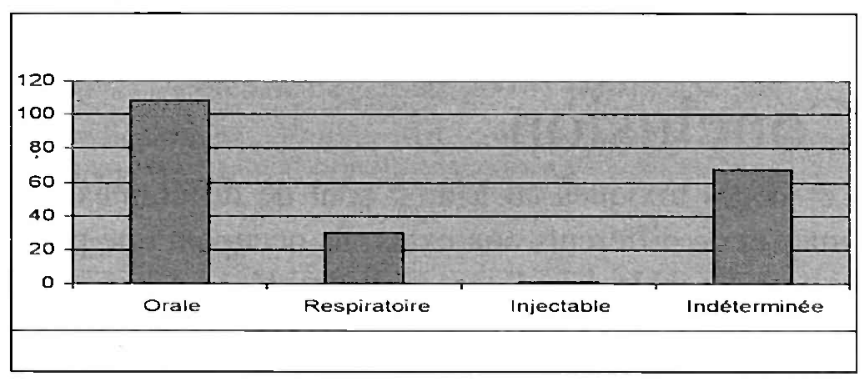

Figure 3 : Fréquence des intoxications mortelles en fonction de la voie d'administration.

La voie respiratoire est liée exclusivement aux intoxications accidentelles alors que la voie orale est plutôt utilisée en cas de suicide.

Presque toutes les villes sont représentées avec un pourcentage élevé dans la ville de Rabat avec 89 cas $(42,4 \%)$, suivie de Casablanca avec 23 cas (11\%), puis Kenitra avec 16 cas $(7,6 \%)$ (Figure 4$)$.

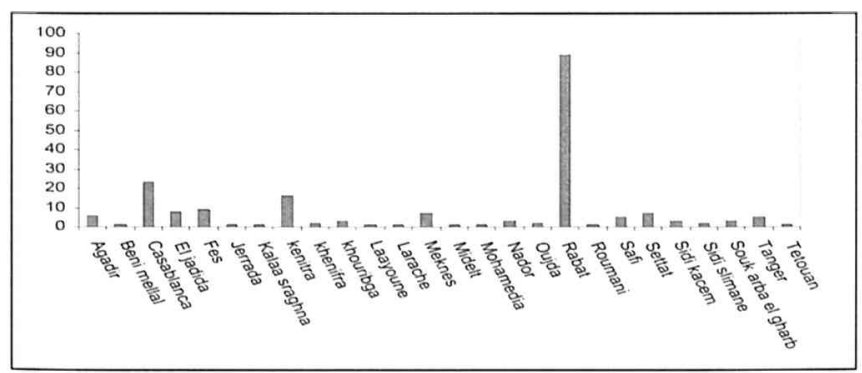

Figure 4 : Fréquence des intoxications mortelles par ville.
L'âge moyen des décès toxiques est de 30 ans \pm 14 . Cependant, toutes les tranches d'âges sont représentées avec un intervalle de 4-80 ans. Les intoxications sont de prédominance masculine avec un pourcentage de $56,5 \%$ contre $43,5 \%$ pour le sexe féminin.

La mortalité dans notre série est élevée quand il s'agit du paraphényldiamine qui vient en tête avec 44 cas $(21,3 \%)$ suivi du monoxyde de carbone avec 35 cas $(16,9 \%)$ et des pesticides 32 cas $(15,5 \%)$ puis le phostoxin avec 20 cas $(9,7 \%)$. L'éthanol est retrouvé dans 24 cas $(11,6 \%)$ (Figure 5).

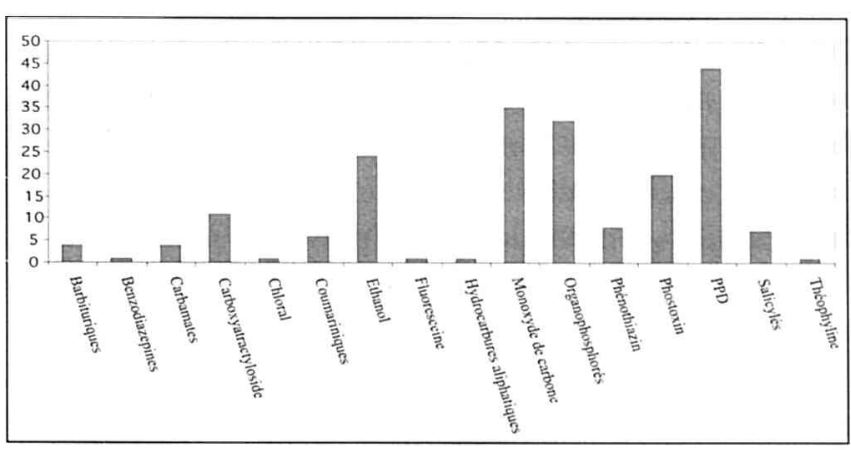

Figure 5 : Fréquence des intoxications mortelles en fonction du toxique incriminé.

\section{Discussion}

Le nombre d'intoxications mortelles de $210(5,9 \%)$ au Maroc pendant la période entre janvier 1999 et décembre 2003 concorde avec d'autres séries $(2,3)$. Cependant, ce chiffre reste sous-estimé du fait du manque d'informations sur les cas de décès toxiques dans les zones rurales, puisque la majorité des prélèvements proviennent de l'axe Casablanca - Kenitra alors que les autres régions du Maroc ne sont pas ou peu représentées.

Nous avons remarqué une diminution de nombre d'intoxications mortelles entre 1999 et 2003 . Ceci pourrait être expliqué par la sensibilisation des gens aux intoxications surtout par le paraphényldiamine et le phostoxin.

Les décès toxiques sont d'origine accidentelle ou suicidaire, les intoxications accidentelles sont essentiellement liées au monoxyde de carbone avec 27 cas $(58,7 \%)$, elles sont de prédominance féminine $(67,4 \%)$ et associées à la voie respiratoire dans 29 cas $(63 \%)$. Les intoxications volontaires sont associées à la voie orale dans $100 \%$ des cas, et au sexe féminin dans 35 cas $(67,3 \%)$, les toxiques incriminés sont essentiellement le paraphényldiamine dans $31,5 \%$ des cas et les pesticides organophosphorés dans $16,7 \%$. Les intoxications mortelles sont fréquentes chez le sujet jeune 
contrairement à ce qui est cité dans d'autres séries (2). Les drogues de prescription sont très présentes dans ces décès, notre série a révélé que les médicaments sont peu impliqués dans les décès toxiques. Ceci pourrait être expliqué par la faible utilisation des médicaments au Maroc et/ou à la bonne prise en charge de ces intoxications au niveau des réanimations, puisque la plupart de ces intoxications médicamenteuses sont urbaines et touchent certaines catégories sociales. Les médicaments décelés sont les benzodiazépines, les salicylés et les neuroleptiques phénothiaziniques.

L'intoxication au monoxyde de carbone reste parmi les intoxications les plus mortelles au Maroc comme c'est le cas en France (4). Ce sont les causes domestiques (chauffe-eau, chauffages d'appoint) qui prédominent, bien avant les accidents professionnels (garage, chaufferie, incendies) et les suicides (5). Dans notre série, les intoxications volontaires sont plus fréquentes que les intoxications accidentelles $(24,2 \%)$, ceci est rencontré dans d'autres séries $(6,7)$. Les pesticides voient leur fréquence augmentée dans les décès toxiques (15\%), ils sont devenus la méthode de choix pour les suicides dans les pays en développement surtout dans les zones rurales et agricoles $(2,8,9,10)$. Les organophosphorés et les carbamates sont les plus représentés $(9,11)$.

Connue depuis 1980 et restée pendant longtemps accidentelle, l'intoxication au paraphényldiamine est devenue actuellement le mode le plus commun et le plus répandu des intoxications volontaires au Maroc comme dans d'autres pays dont l'Inde ou l'incidence de l'intoxication au paraphényldiamine est passée de 0,06 à $0,5 \%$ des admissions de 1981 à 1987 (12), d'autres cas ont été rapportés en Tunisie. L'incidence au Maroc où cette intoxication prend de plus en plus d'ampleur n'est malheureusement pas connue et les premiers cas d'intoxication au paraphényldiamine ont été recensés à la fin des années 1980 (13). Précisément en 1978 et depuis, plusieurs publications se sont succédées (14, 15), Son danger provient de son utilisation par les femmes comme teinture capillaire noire ou adjuvant de henné dans plusieurs pays d'Afrique et de Moyen Orient, comme teinture des fourrures, dans la fabrication d'articles domestiques, d'agents cosmétiques, des pneus, et le développement photographique (16). La mortalité des intoxications à la PPD est très lourde dans toutes les séries, elle varie de 20 à $42 \%$ Elle est liée au retard de consultation dû surtout à l'éloignement géographique par rapport à la structure hospitalière, au bas niveau socioéconomique, Les causes de mortalité sont généralement le syndrome asphyxique à la phase initiale et la rhabdomyolyse et ses complications électrolytiques et rénales à la phase secondaire (13). Le phostoxin ou phosphure d'aluminium et de magnésium est un insecticide et raticide utilisé pour conserver les produits agricoles. Très toxiques pour les mammifères y compris l'Homme. Il a été la cause des suicides au nord de I'Inde (17). Au Maroc, ce produit cause aussi des dégâts, $9,7 \%$ des intoxications dans notre série, malgré les efforts fournis pour réglementer sa vente depuis la ratification de la convention de Rotterdam pour le contrôle du commerce des produits chimiques.

L'alcool est retrouvé dans $11,4 \%$ des cas avec des intoxications accidentelles et volontaires, ce qui est confirmé par d'autres études (18).

Les intoxications par les plantes peuvent apparaître dans deux circonstances bien distinctes, lors de l'ingestion de végétaux frais tenus pour comestibles, ou lors d'automédications par des drogues végétales ou des médicaments en contenant. Dans notre série, la plante retrouvée associée aux décès toxiques est le chardon à glu qui a la particularité d'être une intoxication pédiatrique, accidentelle et rurale, chose qui rend sa prise en charge et sa prévention difficiles.

Il faut signaler enfin que cette étude représente seulement les intoxications aiguës directes et ne prend pas en considération les associations toxiques sinon le nombre de toxiques mortels pourrait être surestimé.

\section{Conclusion}

Les décès toxiques au Maroc sont de différentes origines et de différents contextes, ils occupent une place non négligeable dans l'ensemble des décès. Cependant, leur nombre reste sous estimé pour plusieurs raisons, notamment le manque d'informations sur les cas de décès toxiques dans les zones rurales. Des efforts restent à fournir par les parties concernés pour déterminer la part exacte des décès toxiques dans l'épidémiologie des mortalités au Maroc et pour l'instauration d'un registre de décès. 


\section{Références}

1. Belkhdar. J. In : Pharmacopée marocaine traditionnelle. Médecine arabe ancienne et savoirs populaires. Ibis presse, Paris, 1997.

2. Jonsson A., Holmgren P., Ahlner J. Fatal intoxications in Swedish forensic autopsy material during 1992-2002. Forensic Sci. Int. 2004 ; 143 : 53-59.

3. Vougiouklakis T., Vassiliki A-B., Mitselou A. Fatal poisoning in the region of Epirus, Greece, during the period 1998-2004. J. Clin. Forensic Med. 2006 ; 13 : 321-325

4. Malbosc R. Intoxications aiguës et chronique par le monoxyde de carbone : aspect analytique et interprétation des oxycarbonémies. Rev. Fr. Lab. 2000 ; 323 : 19 25.

5. Actualité de l'intoxication au monoxyde de carbone Rev. Fr. Lab., 2000, $327: 24$

6. Abdollahi M., Jalali N., Sabzevari O., Hoseini R., Ghane T -A. Retrospective study of poisoning in Tehran. J. Toxicol. Clin. Toxicol. $1997 ; 35: 387-393$.

7. Afshari R., Majdzadeh R., Balali-mood M. Pattern of acute poisoning in Mashhad, Iran, 1993-2000. J. Toxicol. Clin. Toxicol. 2004 ; 42 : 965-973.

8. Marques E.G.P. Acute intoxication by azinphos-ethyl. J. Anal.Toxicol. 1990 ; 14 : 243-246.

9. Kambiz S., Faryadi M., Sardari F. Acute pesticide poisoning related deaths in Tehran during the period 20032004. J. Forensic Leg. Med. 2007 ; 14(6) : 352-354.

10. Eddleston M., Philips MR. Self poisoning with pesticides. Br. Med. J. 2004 ; 328 : 42-44.
11. Baudet M., Amouroux N., Houin G. Intoxications Accidentelles domestiques. Rev. Fr. Labor. 2000 ; 323 : 45-49.

12. Chug KS., Malik GH., Singhal PC. Acute renal failure following paraphenylen diamine (hair dye) poisoning: report of two cases. J Med. 1980 ; 13(1-2) : 131-137.

13. Idrissi M- H., Oualili L., Abidi K., Abouqal R., Kerkeb O., Zeggwagh A A. Facteurs de gravité de l'intoxication aiguë au phosphure d'aluminium (Phostoxinß). Ann. Fr. Anesth. Réanim. $2006 ; 25: 382-385$.

14. Bourkia A, Jabrane A-J., Ramdani B., Zaid D. Toxicité systémique de la PPD, Presse Med. $1988 ; 17: 125$.

15.Zeggwagh A., Abouqal R., Madani N. Myocardite toxique due à l'intoxication au paraphénylène-diamine. À propos de deux cas, Reanim. Urg. 1996 ; 5 : 699-703.

16. Motaouakkil S., Charra B., Hachimi A., Ezzouine H., Guedari H., Nejmi H., Benslama A. Rhabdomyolyse et intoxication à la paraphénylène-diamine. Ann. Fr. Anesth. Réanim. 2006 ; 25 (7) : 708-713.

17. Ching-Hung H., Bei-Ching C., Liu M., Jih-Heng L., Chiou-Jong C., Ruey-Yu C. Phosphine-induced oxidative damage in rats: role of glutathione. Toxicology. 2002 ; 179(1-2): 1-8.

18. Drossch D.M., Rich CL., Isacsson G. Psychoactive substances in suicides. Comparison of toxicological finding in two samples. Am.J. Forensic Med. Pathol. 2001 ; 22 : 239-243. 